\title{
EHHM, a novel phenolic natural product from Livistona chinensis, induces autophagy-related apoptosis in hepatocellular carcinoma cells
}

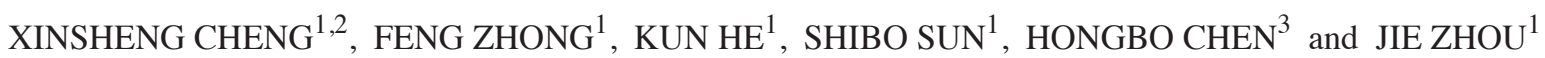 \\ ${ }^{1}$ Department of Hepatobiliary Surgery, Nanfang Hospital, Southern Medical University, Guangzhou, Guangdong 510515; \\ ${ }^{2}$ Department of Hepatobiliary Surgery, Nanshan Affiliated Hospital of Shenzhen University, \\ Shenzhen, Guangdong 518000; ${ }^{3}$ The Shenzhen Key Laboratory of Gene and Antibody Therapy, \\ Graduate School at Shenzhen, Tsinghua University, Shenzhen, Guangdong 518055, P.R. China
}

Received May 10, 2015; Accepted August 19, 2016

DOI: $10.3892 / \mathrm{ol} .2016 .5178$

\begin{abstract}
Hepatocellular carcinoma (HCC) ranks the second cause of cancer-associated mortality worldwide. In the present study, the effects and mechanisms of a new phenolic natural product E-[6'-(5'-hydroxypentyl) tricosyl]-4-hydroxy-3-methoxycinnamate (EHHM) isolated from Livistona chinensis on the growth of HCC cells were investigated. It was observed that EHHM treatment significantly suppressed cell proliferation and colony formation, and induced cell apoptosis via a mitochondria-dependent caspase pathway in HepG2 cells in a time- and dose-dependent manner. Meanwhile, EHHM treatment also led to upregulated expression of autophagy protein 5 (Atg5), Beclin 1 and light chain 3 (LC3)-II proteins, and accumulation of green fluorescent protein-LC3 punctate florescent foci in HCC cells, suggesting that EHHM-induced apoptosis is accompanied by autophagy induction. Western blotting revealed that EHHM-induced autophagy is related to the inhibition of the Akt/mechanistic target of rapamycin/p70 ribosomal protein S6 kinase signaling pathway. Furthermore, treatment with Atg5 small interfering RNA or autophagy inhibitors significantly enhanced EHHM-mediated growth inhibition and apoptotic cell death, indicating that autophagy serves as a self-protective mechanism in EHHM-treated HCC cells, and that combined
\end{abstract}

Correspondence to: Mr. Jie Zhou, Department of Hepatobiliary Surgery, Nanfang Hospital, Southern Medical University, 1838 Guangzhou North Avenue, Guangzhou, Guangdong 510515, P.R. China

E-mail: zhouj20@sina.com

Mr. Hongbo Chen, The Shenzhen Key Laboratory of Gene and Antibody Therapy, Graduate School at Shenzhen, Tsinghua University, 2279 Lishui Road, Shenzhen, Guangdong 518055, P.R. China

E-mail: chen.hongbo@sz.tsinghua.edu.cn

Key words: EHHM, hepatocellular carcinoma, apoptosis, autophagy treatment with EHHM and autophagy inhibitors may be an effective therapeutic strategy for HCC.

\section{Introduction}

Hepatocellular carcinoma (HCC) is the most frequently diagnosed cancer and the second major cause of cancer mortality worldwide (1-3). Infection with hepatitis B or C virus is the leading cause of HCC (4). Surgical resection is mostly selected in HCC treatment, followed by radiation therapy and chemotherapy; however, the 5-year survival rates following resection are very low, and the recurrence rates, mainly due to primary tumor metastasis and formation of new tumors, exceed $70 \%(5,6)$. Therefore, it is necessary and urgent to develop new effective chemotherapeutic agents for the treatment of HCC.

Livistona chinensis, a species of subtropical palm tree in eastern Asia, is widely distributed and cultivated in tropical and temperate regions, including several islands in the South China Sea, Southern Japan and Taiwan (7). The extract from the fruit of L. chinensis has been shown to exhibit antibacterial activity (8), and was traditionally used for folk remedy for various tumors, including chorionic carcinoma, esophageal carcinoma, leukemia and nasopharyngeal carcinoma $(9,10)$. E-[6'-(5'-hydroxypentyl) tricosyl]-4-hydroxy-3-methoxycinnamate (EHHM), a new phenolic compound isolated from the L. chinensis fruit, has been shown to have antioxidant activity and a potent anticancer activity by inhibiting cancer cell proliferation $(9,11)$. However, the antitumor effects and possible mechanisms of EHHM against human HCC have not yet been investigated. In the present study, it was demonstrated that EHHM can inhibit the proliferation and induce the apoptosis of HCC cells. In addition, EHHM induced autophagy in HCC cells.

The induction of cancer cell apoptosis is the main consideration in anticancer compound research (12-15). Cell morphology shrinkage, nuclear fragmentation, chromatin condensation and chromosomal DNA fragmentation are typically observed during apoptosis (16). However, to escape from apoptosis, cancer cells may also trigger various other pathways 
such as autophagy in order to overcome the adverse environment (17-19).

Autophagy is an intracellular degradation process, through which, a portion of the cytoplasmic components is delivered to lysosomes to be degraded (20). The cell fates are different when autophagy is induced in response to multiple environmental stresses (20). Autophagy can induce cell death (21) or serve as a survival mechanism that protects tumor cells from apoptotic death $(22,23)$. Meanwhile, several studies have suggested that, in certain circumstances, inhibition of protective autophagy can promote cell apoptosis (24). Therefore, autophagy may be a novel approach for cancer treatment. In the present study, it was observed that EHHM induced cytoprotective autophagy through the Akt/mechanistic target of rapamycin (mTOR) signaling pathway in HCC cells, and that inhibition of autophagy by small interfering RNA (siRNA) targeting autophagy-related genes or autophagy inhibitors could lead to the enhancement of EHHM-mediated cytotoxicity through increasing cell apoptosis in vitro and in vivo.

\section{Materials and methods}

Reagents. EHHM was isolated and identified as described in a previous study (9). EHHM was dissolved in dimethyl sulfoxide (DMSO) at a stock solution of $50 \mathrm{mM}$, stored at $-20^{\circ} \mathrm{C}$ and diluted with culture medium prior to each experiment. The human HCC cell line HepG2, which was obtained from the American Type Culture Collection (Manassas, VA, USA), was routinely maintained in Dulbecco's modified Eagle medium (DMEM; Gibco; Thermo Fisher Scientific, Inc., Waltham, MA, USA) with $10 \%$ fetal calf serum (Gibco; Thermo Fisher Scientific, Inc.) and incubated in a humidified atmosphere with $5 \% \mathrm{CO}_{2}$ at $37^{\circ} \mathrm{C}$. 3-(4,5-Dimethylthiazol-2-yl)2,5-diphenyltetrazolium bromide (MTT) was purchased from Roche Diagnostics (Indianapolis, IN, USA). Trypan blue was purchased from Sangon Biotech Co., Ltd. (Shanghai, China). The pEGFPc1-LC3 plasmid was a gift from Professor Marja Jäättelä (Institute of Cancer Biology, Danish Cancer Society, Copenhagen, Denmark) (25). Benzyloxycarbonyl-Val-Ala-Asp (OMe) fluoromethylketone (Z-VAD-FMK) was purchased from Sigma-Aldrich (Merck Millipore, Darmstadt, Germany). Lipofectamine 2000 and FITC Annexin V/Dead Cell Apoptosis kit were purchased from Invitrogen (Thermo Fisher Scientific, Inc.). JC-1 Mitochondrial Membrane Potential Assay kit was purchased from Nanjing KeyGen Biotech Co., Ltd. (Nanjing, China). 4',6-Diamidino-2-phenylindole and Hoechst 33258 were obtained from Sangon Biotech Co., Ltd. Bafilomycin A1 (Baf A1) and 3-methyl adenine (3-MA) were purchased from Sigma-Aldrich (Merck Millipore). ECL Western Blotting Substrate kit was purchased from Abcam (Cambridge, MA, USA). Antibodies were purchased from the following suppliers: Anti- $\beta$-actin antibody (cat.no. AF0003) from Beyotime Institute of Biotechnology (Haimen, China); anti-caspase 3 antibodies (cat. no. ab13585) from Abcam; and anti-light chain 3 (LC3; cat. no. 3868), anti-autophagy protein 5 (Atg5; cat. no. 12994) and anti-Beclin 1 (cat. no. 3495) antibodies from Cell Signaling Technology, Inc. (Danvers, MA, USA).

Cell proliferation and viability assays. Cell proliferation was determined by MTT assay as described previously (26).
Briefly, $90 \mu \mathrm{l}$ cells ( $10^{5}$ cells) were seeded into a 96-well plate and precultured for $24 \mathrm{~h}$, prior to be treated with EHHM for 24 or $48 \mathrm{~h}$. Next, $10 \mu \mathrm{l}$ of MTT $(5 \mathrm{mg} / \mathrm{ml})$ was added to each well and incubated for additional $4 \mathrm{~h}$ at $37^{\circ} \mathrm{C}$. The medium was removed carefully, and $150 \mu 1$ of DMSO was added into the plate, and then the 96 -well plate was agitated for $10 \mathrm{~min}$. The absorbance (A) was measured at $570 \mathrm{~nm}$ by an automated microplate reader (BioTek Instruments, Inc., Winooski, VT, USA). The cell death rate was calculated as follows: Cell death $(\%)=\left(\right.$ mean $\mathrm{A}_{570}$ of the control group - mean $\mathrm{A}_{570}$ of the experimental group)/(mean $\mathrm{A}_{570}$ of the control group - mean $\mathrm{A}_{570}$ of the blank group) $\mathrm{x} 100 \%$. Cell viability was estimated by trypan blue dye exclusion (27).

Soft agar colony formation assay. Colony formation in soft agar was tested by suspending $1 \times 10^{3}$ cells in $1 \mathrm{ml}$ of DMEM with $0.3 \%$ low melting point agarose (Merck Millipore) in the presence of different concentrations of EHHM, and then plating them on a bottom layer containing $0.6 \%$ agarose and $10 \%$ fetal bovine serum (Gibco; Thermo Fisher Scientific, Inc.) in a 6-well plate in triplicate. After 15 days, the plates were stained with $0.1 \%$ gentian violet, and the colonies were counted under a light microscope $(28,29)$.

Nuclei morphology observation by Hoechst staining. Cell morphological changes in the nucleus were determined by Hoechst 33258 staining as described previously (30). Briefly, cells treated with different concentrations of EHHM were fixed with $4 \%$ polyoxymethylene for $10 \mathrm{~min}$ and then stained with Hoechst $33258(1 \mathrm{mg} / \mathrm{ml})$ at room temperature in the dark for $5 \mathrm{~min}$. Morphological changes of nuclei were observed and imaged using a fluorescence microscope with an excitation wavelength of 330-380 nm. Cells with round nuclear morphology were considered normal, while those with condensed/fragmented and bright nuclei were regarded as apoptotic.

Analysis of cell proliferation and apoptosis. Cells were treated with EHHM, 3-MA or Baf A1 for the indicated concentrations and time points. Apoptotic cells were analyzed using annexin V-fluorescein isothiocyanate (FITC) /propidium iodide (PI) double staining methods as described previously (31). Briefly, HepG2 cells were treated with the indicated concentrations of EHHM in the presence or absence of 3-MA (5 mM) or Baf A1 (10 nM) for $24 \mathrm{~h}$. Cells were digested into a single-cell suspension with ehylenediaminetetraacetic acid-free trypsin and stained according to the manufucturer's protocol of the FITC Annexin V/Dead Cell Apoptosis kit. The stained cells were immediately analyzed by flow cytometry. Cells undergoing early-stage apoptosis are stained with annexin V-FITC only (green fluorescence), while cells at late-stage apoptosis and necrotic cells are stained with both annexin V-FITC and PI (both green and red fluorescence) (32). In addition, to determine whether EHHM-induced apoptosis is caspase-dependent, HepG2 cells were pretreated with $20 \mathrm{mM}$ Z-VAD-FMK for $1 \mathrm{~h}$ followed by treatment with $15 \mu \mathrm{M}$ EHHM for $24 \mathrm{~h}$, and the inhibition rate was determined by MTT assay, as described previously.

Transfection of cells with siRNA. siRNA targeting human Atg5 (5'-CCUUUGGCCUAAGAAGAAATTdTdT-3') was 
synthesized by Shanghai GenePharma Co., Ltd. (Shanghai, China), and a nonspecific siRNA served as a negative control. Cells seeded in $60-\mathrm{mm}$ dishes were transfected with siRNAs for $36 \mathrm{~h}$ prior to treatment with EHHM.

JC-1 mitochondrial membrane potential detection. Cell mitochondrial membrane potential changes were analyzed by JC-1 staining as described previously (33). Cells were incubated with or without EHHM for $24 \mathrm{~h}$, followed by incubation with JC-1 working solution, and then the cells were incubated in $5 \% \mathrm{CO}_{2}$ for $20 \mathrm{~min}$. Cells was washed twice with JC-1 incubation buffer, and then the cells labeled with JC-1 were detected with a fluorescence microscope. JC-1 can selectively enter the intact mitochondria with high membrane potential and form polymers to emit red fluorescence, whereas in mitochondria with low membrane potential, it remains in the monomeric form in the cytosol and emits a green fluorescence (16).

Western blotting. Cells were treated with different concentrations of EHHM in the presence or absence of 3-MA $(5 \mathrm{mM})$ or Baf A1 $(10 \mathrm{nM})$ for $24 \mathrm{~h}$, and then lysed in lysis buffer [50 mM Tris-HCl (pH 6.8), $2 \%$ w/v sodium dodecyl sulfate (SDS), $10 \%$ glycerol, $10 \mathrm{mM}$ dithiothreitol, $1 \mathrm{mM}$ phenylmethane sulfonyl fluoride and 1X protease inhibitor cocktail (Roche, Basel, Switzerland)]. Equal amounts of protein samples were quantified using the RC DC ${ }^{\mathrm{TM}}$ Protein Assay Kit I (Bio-Rad Laboratories, Hercules, CA, USA) and electrophoresed by $8-12 \%$ SDS-polyacrylamide gel electrophoresis and transferred to a polyvinylidene fluoride membrane. The membranes were blocked with a 5\% non-fat milk powder solution in Tris-buffered saline with Tween 20 (TBST) buffer [50 mM Tris- $\mathrm{HCl}(\mathrm{pH} 7.6), 150 \mathrm{mM} \mathrm{NaCl}$ and $0.05 \%$ Tween 20 ] for $1 \mathrm{~h}$ at room temperature and then incubated with the corresponding primary antibodies $(1: 1,000)$ overnight at $4^{\circ} \mathrm{C}$. Upon washing with TBST, membranes were incubated with horseradish peroxidase-conjugated anti-mouse (cat. no. 31430; 1:5,000) and anti-rabbit (cat. no. 31460; 1:5,000) IgG secondary antibodies (Thermo Fisher Scientific, Inc.) for $2 \mathrm{~h}$ at room temperature and detected with ECL Western Blotting Substrate kit (Thermo Fisher Scientific, Inc.) (34).

Visualization of cells with green fluorescent protein $(G F P)-L C 3$ vesicles. Cells plated in 6-well plates were transfected with the pEGFPc1-LC3 plasmid using Lipofectamine 2000 . After $24 \mathrm{~h}$ of transfection, cells were treated with different concentrations of EHHM in the presence or absence of autophagy inhibitors, 3-MA $(5 \mathrm{mM})$ or Baf A1 $(10 \mathrm{nM})$, for $12 \mathrm{~h}$. The patterns of GFP-labeled LC3 in transfected cells were examined and imaged by fluorescence microscopy.

HCC xenograft experiments. A total of 24 female 6-7 week-old nude immunodeficient mice (nu/nu) (15-20 g) were purchased from the Guangdong Province Medical Animal Center (Guangzhou, China) and bred in the laboratory animal facility of Tsinghua University (Shenzhen, China). The mice were housed at $25^{\circ} \mathrm{C}$ and exposed to $12 \mathrm{~h} \mathrm{light/dark}$ cycles with free access to food and water. All animal studies were approved by the Institutional Animal Care and Use
Committee of Tsinghua University. The mice were subcutaneously injected with HepG2 cells $\left(2.0 \times 10^{6}\right.$ cells) in $100 \mu \mathrm{l}$ of DMEM without serum. The mice were randomly divided into four groups (6 mice/group) when the tumors reached a palpable size and then treated with vehicle control, 3-MA $(24 \mathrm{mg} / \mathrm{kg})$ and EHHM $(50 \mathrm{mg} / \mathrm{kg})$, alone or in combination with 3-MA, every 2 days. Vernier caliper measurements of the longest perpendicular tumor diameters were performed every 2 days to estimate the tumor volume with the following formula: $4 \pi / 3 \times(\text { width } / 2)^{2} \times($ length $/ 2)$, which represents the three-dimensional volume of an ellipsoidal tumor tissue (35).

Statistical analysis. Data from three independent experiments were expressed as the mean \pm standard deviation, unless otherwise noted. The Student's $t$ test was used to evaluate the variables in the present study. $\mathrm{P}<0.05$ was considered to indicate a statistically significant difference.

\section{Results}

Proliferation-inhibition and apoptosis-induction effect of EHHM on HepG2 cells. EHHM is a novel plant metabolite isolated from the fruit of $L$. chinensis (Fig. 1A). In the present study, the cytotoxicity of EHHM towards human HepG2 cells was examined. By MTT assay, it was demonstrated that EHHM had a moderate cytotoxicity towards HepG2 cells, with a half maximal inhibitory concentration of $41.62 \pm 4.57 \mu \mathrm{M}$ (Fig. 1B). Meanwhile, using trypan blue dye exclusion analysis, it was demonstrated that EHHM inhibited the proliferation and growth of HepG2 cells in a time- and dose-dependent manner (Fig. 1C). Next, the effect of EHHM on colony formation was tested using soft agar colony formation assays. The results revealed that the clonogenic activity of HepG2 cells was significantly suppressed (Fig. 1D and E). These results suggested that EHHM can significantly inhibit the proliferation and growth of HCC cells in a time- and dose-dependent manner.

EHHM induces apoptosis of HCC cells via a mitochondriadependent caspase activation cascade. It was tested whether EHHM can induce the apoptosis of HCC cells. By Hoechst 33258 staining, HepG2 cells treated with the indicated concentrations of EHHM exhibited chromatin condensation and fragmentation, which are typical apoptotic nuclear morphological changes (Fig. 2A). Meanwhile, flow cytometry assay using annexin V/PI staining also confirmed that treatment with EHHM induced apoptosis in HepG2 cells in a time- and dose-dependent manner (Fig. 2B). Caspase 3, a critical executor of apoptosis, is either partially or totally responsible for the proteolytic cleavage of numerous key proteins such as the nuclear enzyme poly (ADP-ribose) polymerase (PARP) (36). The inactive full-length caspase 3 is processed during apoptosis, generating an activated $17-\mathrm{kDa}$ fragment termed cleaved caspase 3 (37). In the present study, both cleaved caspase 3 and cleaved PARP were detected subsequent to EHHM treatment at different concentrations $(15,45,90$ and $150 \mu \mathrm{M})$, and a reduction in pro-caspase 3 (through cleavage) was observed (Fig. 2C). To further confirm that EHHM-induced cell apoptosis is caspase-dependent, HepG2 cells were pretreated with Z-VAD-FMK (20 mM), a pan-caspase inhibitor, for $1 \mathrm{~h}$ and then treated with $15 \mu \mathrm{M}$ EHHM for additional $24 \mathrm{~h}$. As 
A<smiles>C=CCC(CCCCCO)CCCCCOC(=O)/C=C/c1ccc(O)c(OC)c1</smiles>

C

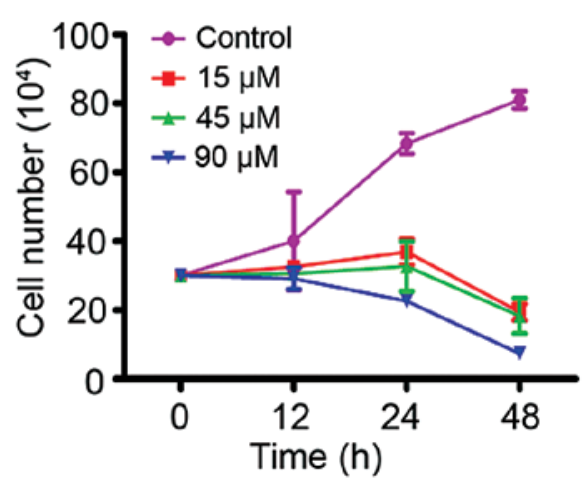

D

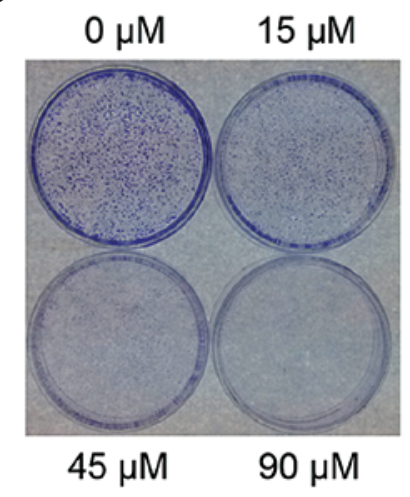

B
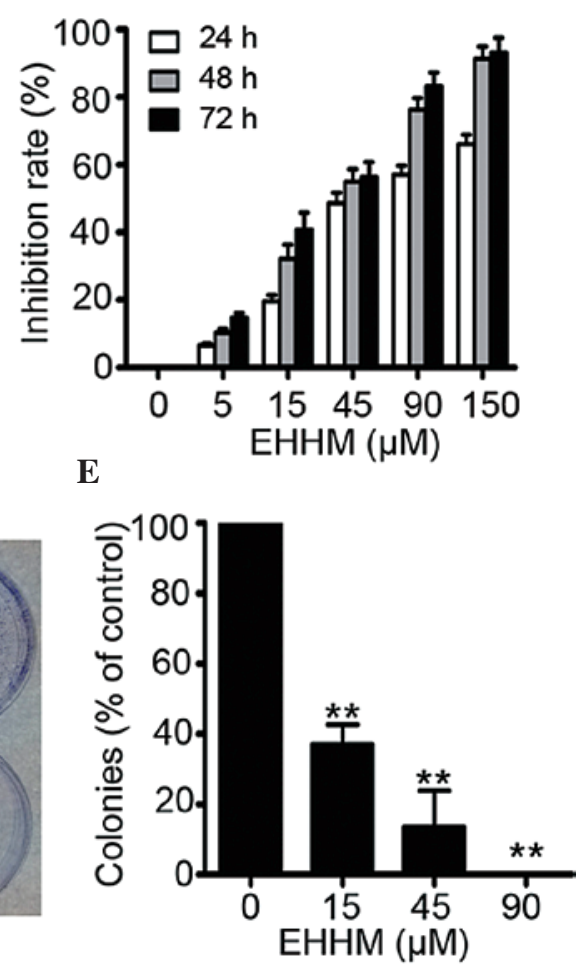

Figure 1. Inhibitory effects of EHHM on HepG2 cells. (A) Chemical structure of EHHM. (B) The inhibitory effects of EHHM on HepG2 cells were analyzed by 3-(4,5-dimethylthiazol-2-yl)-2,5-diphenyltetrazolium bromide assay. (C) The inhibitory effects of EHHM on the viability of HepG2 cells were analyzed by trypan blue exclusion assay. (D) HepG2 cells were treated with EHHM at the indicated concentrations, and colony formation was observed. (E) The colonies were counted, and the data were expressed as a percentage of the control group ( $0-\mu \mathrm{M}$ EHHM treatment). The experiment was conducted in triplicate, and the mean \pm standard deviation was calculated $\left({ }^{* *} \mathrm{P}<0.01\right)$. EHHM, E-[6'-(5'-hydroxypentyl)tricosyl]-4-hydroxy-3-methoxycinnamate.

shown in Fig. 2D, EHHM-induced apoptosis was significantly suppressed. Since the activation of caspase 3/caspase 9 involves mitochondria permeability changes and the release of cytochrome $c$, the present study next examined changes in the mitochondrial transmembrane potential following EHHM treatment. As shown in Fig. 2E, in EHHM-treated HepG2 cells, JC-1 remained in the cytoplasm in its monomeric form and emitted green fluorescence, whereas in the cells without EHHM treatment, JC-1 accumulates and aggregates in the mitochondria, emitting red fluorescence. This result suggested that EHHM treatment damaged the mitochondrial transmembrane of HepG2 cells. Meanwhile, cytochrome $c$ release from mitochondria was also investigated. As shown in Fig. 2C, a significant increase in released cytochrome $c$ was detected at $24 \mathrm{~h}$ after $15-\mu \mathrm{M}$ EHHM treatment.

Overall, these results suggested that EHHM treatment significantly suppressed HepG2 proliferation and induced apoptosis in a time- and dose-dependent manner, and that EHHM-induced apoptosis in HepG2 cells is mediated via mitochondria-dependent caspase cascade activation.

EHHM induces Akt/mTOR signaling-related autophagy in HepG2 cells. Notably, several vacuoles were observed in EHHM-treated HepG 2 cells, but not in untreated cells, by light microscopy, indicating that EHHM treatment may be associated with cell autophagy.

Autophagy, a cellular pathway involved in protein and organelle degradation, plays complicated roles in cancer development (38). During the autophagy process, LC3-I is converted into LC3-II via a ubiquitin-like system involving Atg7 and Atg3, which promotes LC3-II relocalization to autophagic vesicles (39). The conversion of LC3-I into LC3-II and the presence of LC3-II in autophagosomes have been used as indicators of autophagy activation (40). To test whether EHHM induces autophagy formation, HepG2 cells transfected with GFP-LC3 were treated with EHHM, and autophagic vacuoles were detected. As shown in Fig. 3A, the number of autophagic vacuoles with a punctate staining pattern of GFP-LC3-II was significantly increased in EHHM-treated cells compared with untreated cells. Accordingly, the protein levels of LC3-II were also significantly increased in HepG2 cells treated with EHHM in a dose-dependent manner (Fig. 3B). In addition, several studies have demonstrated that Beclin 1 participates in the initiation of the autophagosome formation, and that Atg5, an E3 ubiquitin ligase, is important in autophagosome elongation $(41,42)$. Therefore, the expression levels of these two proteins were also investigated in EHHM-treated cells. As shown in Fig. 3B, the expression levels of Atg5 and Beclin 1 proteins in cells exposed to EHHM were also increased in a dose-dependent manner.

The phosphatidylinositol 3-phosphate kinase (PI3K)/Akt/ p70 ribosomal protein S6 kinase (p70S6K) signaling pathway is known to regulate autophagy, growth, proliferation and survival in response to a variety of stresses in mammalian cells (43). To investigate whether the Akt/mTOR/p70S6K signaling pathway was involved in EHHM-induced apoptosis 
A

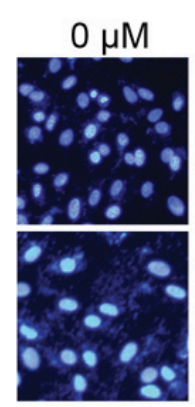

$45 \mu \mathrm{M}$
B

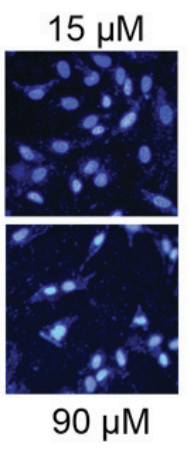

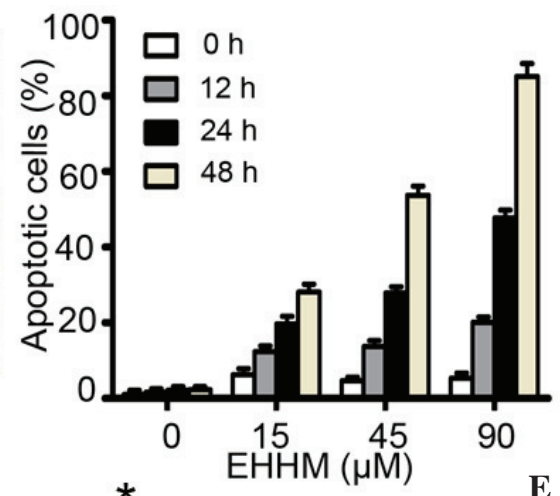

E

C

$\operatorname{EHHM}(\mu \mathrm{M}) \quad 0 \quad 15 \quad 45 \quad 90 \quad 150$

Cytosol-Cytochrome C _..

Pro-caspase 3

Cleaved-caspase 3

Cleaved-caspase9

PARP

Cleaved-PARP

$\beta$-actin
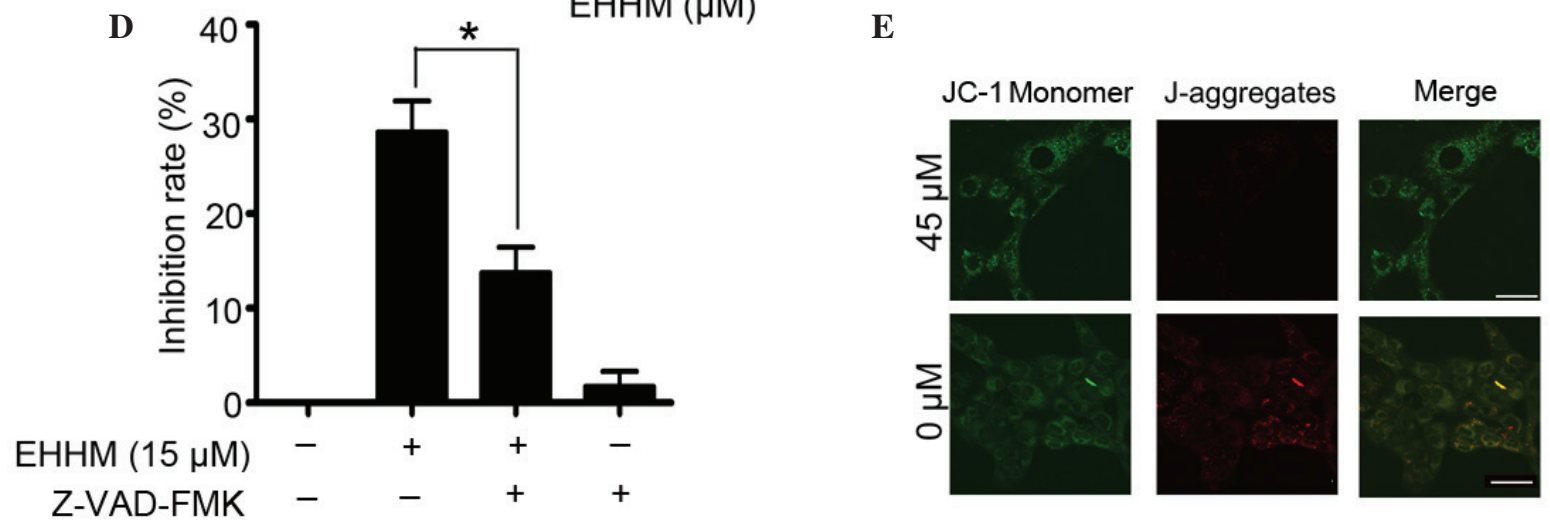

Figure 2. EHHM induces the apoptosis of HepG2 cells via a mitochondria-dependent caspase activation cascade. (A) HepG2 cells were treated with EHHM for $24 \mathrm{~h}$, and evaluated by Hoechst 33258 assay. Scale bar, $50 \mu \mathrm{m}$. (B) HepG2 cells were treated with EHHM for $24 \mathrm{~h}$, and the apoptotic cell death rate was analyzed by annexin V/propidium iodide staining and flow cytometry. (C) HepG2 cells were treated with EHHM (15 $\mu$ M), and western blotting was performed using the indicated antibodies. (D) HepG2 cells were pretreated with Z-VAD-FMK $(20 \mathrm{mM})$ for $1 \mathrm{~h}$ and then treated with EHHM at $15 \mu \mathrm{M}$ for $24 \mathrm{~h}$, and the inhibition rate was determined by 3-(4,5-dimethylthiazol-2-yl)-2,5-diphenyltetrazolium bromide assay (" $\mathrm{P}<0.05)$. (E) The mitochondrial membrane potential of HepG2 cells treated with EHHM at the indicated concentration for $24 \mathrm{~h}$ was determined by JC-1 staining. Scale bar, $20 \mu \mathrm{m}$. Data represents the mean \pm standard deviation of three independent experiments. EHHM, E-[6'-(5'-hydroxypentyl)tricosyl]-4-hydroxy-3-methoxycinnamate; FITC, fluorescein isothiocyanate; PARP, poly (ADP-ribose) polymerase; $\mathrm{Cy}$, cyanine.

A

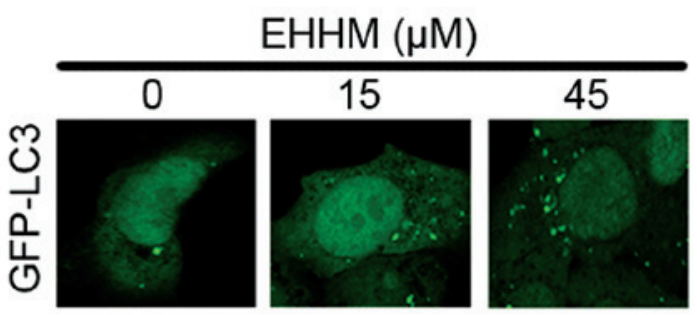

C

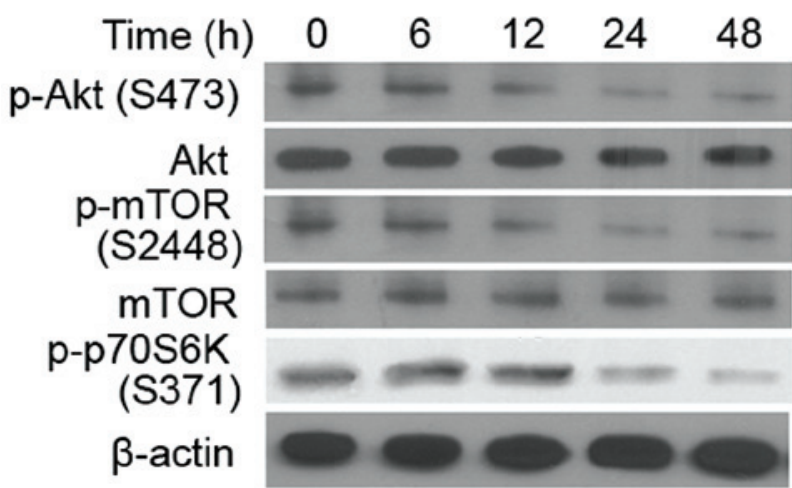

B

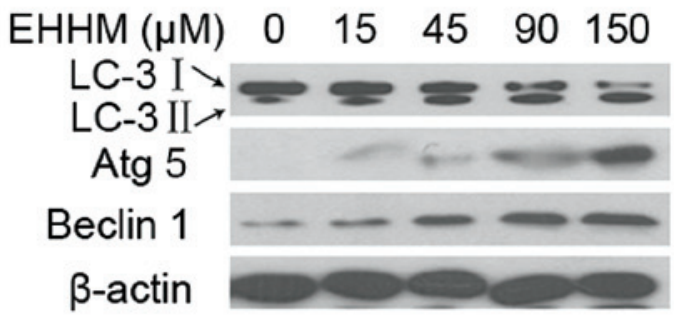

D

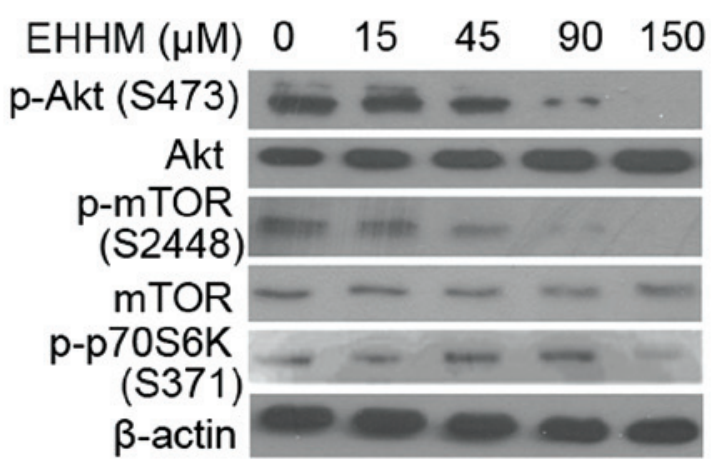

Figure 3. Autophagy is triggered by EHHM in HepG2 cells. (A) HepG2 cells were transfected with a GFP-LC-3-expressing plasmid for 24 h, and then treated with EHHM at the indicated concentrations for additional $24 \mathrm{~h}$. The cells were then observed under a confocal microscope. Scale bar, $10 \mu \mathrm{m}$. (B) HepG2 cells were treated with EHHM at the indicated concentrations for $24 \mathrm{~h}$, and western blotting was performed with antibodies against autophagy marker proteins. (C and D) EHHM treatment inhibited the AKT/mTOR/p70S6K signaling pathway in a (C) time- and (D) dose-dependent manner. EHHM, E-[6'-(5'-hydroxypentyl)tricosyl]-4-hydroxy-3-methoxycinnamate; GFP, green fluorescent protein; LC3, light chain 3; Atg5, autophagy protein 5; p-, phosphorylated; mTOR, mechanistic target of rapamycin; p70S6K, p70 ribosomal protein S6 kinase. 
A

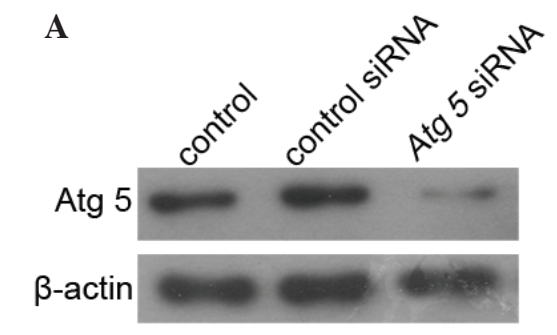

$\mathbf{C}$

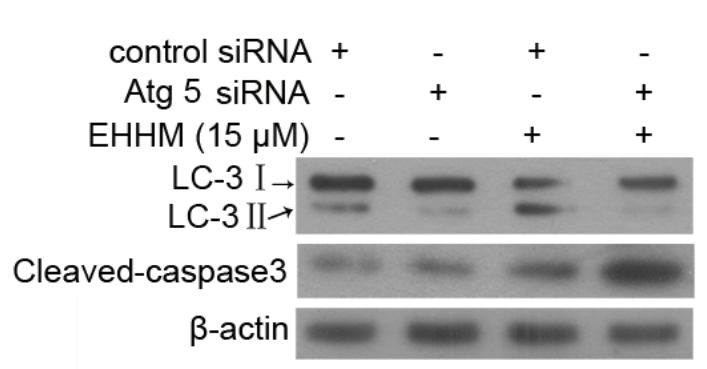

B

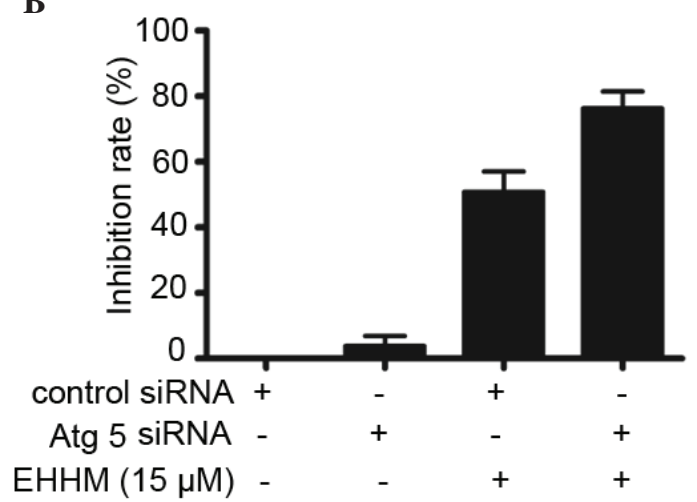

D

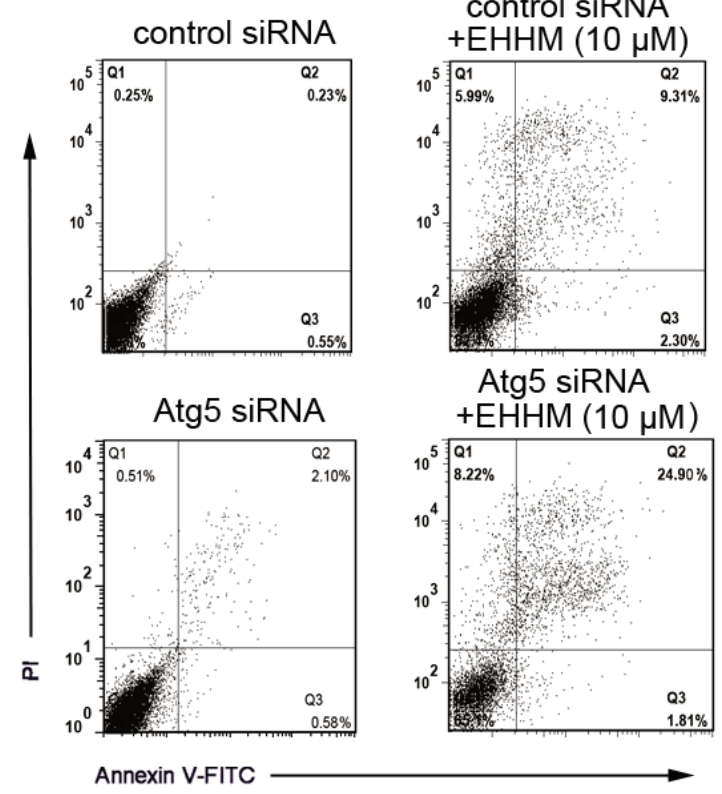

Figure 4. Knockdown of Atg5 inhibits autophagy but enhances EHHM-induced apoptosis in HepG2 cells. (A) Western blot analysis of Atg5 expression in HepG2 cells with the indicated treatment. (B) HepG2 cells were treated with control siRNA or Atg5 siRNA, with or without EHHM (15 $\mu$ M) for 24 h. The inhibition rate was determined by 3-(4,5-dimethylthiazol-2-yl)-2,5-diphenyltetrazolium bromide assay. (C) Western blot analysis of LC3-I/II and caspase 3 activation of HepG2 cells with the indicated treatments. (D) The apoptosis of HepG2 cells subjected to the indicated treatments was analyzed by flow cytometry. EHHM, E-[6'-(5'-hydroxypentyl)tricosyl]-4-hydroxy-3-methoxycinnamate; LC3, light chain 3; Atg5, autophagy protein 5; siRNA, small interfering RNA; FITC, fluorescein isothiocyanate; PI, propidium iodide.

and autophagy, western blotting was performed to detect the expression levels of the associated proteins. As shown in Fig. 3C and D, EHHM inhibited the phosphorylation of Akt, mTOR and p70S6K in a time- and dose-dependent manner.

Taken together, these data indicated that EHHM was able to induce autophagy in HepG2 cells, which involves the $\mathrm{Akt} / \mathrm{mTOR} / \mathrm{p} 70 \mathrm{~S} 6 \mathrm{~K}$ signaling pathway.

Autophagy inhibition enhances the cell growth-inhibition and apoptotic effect of EHHM. As demonstrated above, EHHM treatment induces cell apoptosis and autophagy in HepG2 cells. As previously reported, autophagy may serve different roles in response to different cellular stresses, protecting or killing the cells (44). To identify which type of autophagy is induced by EHHM, HepG2 cells were transfected with Atg5 siRNA (Fig. 4A), and the proliferation effect of EHHM on HepG2 cells was evaluated using an MTT assay. As illustrated in Fig. 4B, knockdown of Atg5 expression significantly enhanced the inhibition effect of EHHM on HepG2 cells compared with control siRNA. Simultaneously, knockdown of Atg5 resulted in the decrease of LC3-I/II conversion and the increase in cleaved caspase 3 expression (Fig. 4C). A flow cytometry assay further confirmed the above results. As shown in Fig. 4D, knockdown of Atg5 significantly enhanced EHHM-induced apoptosis of HepG2 cells.

Furthermore, the effects of autophagy specific inhibitors (3-MA and Baf A1) on EHHM-induced growth inhibition and apoptosis were investigated. 3-MA inhibits autophagy via blocking the autophagosome formation in the early stage of autophagosome formation (45), whereas Baf A1, a macrolide antibiotic derived from Streptomyces griseus, inhibits autophagy by blocking the fusion of autophagosomes with lysosome in the late stage of autophagy (46). As shown in Fig. 5A, the EHHM-induced upregulation of Atg5 was inhibited by 3-MA and Baf A1 treatment. As expected, the accumulation of GFP-LC3-II on autophagic vacuoles induced by EHHM 
A

B

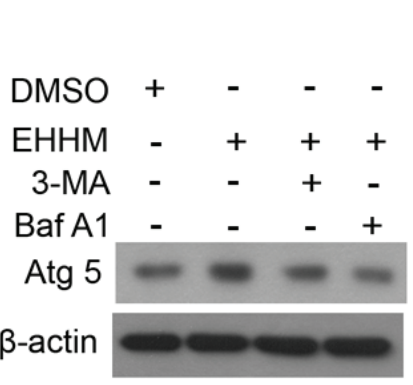

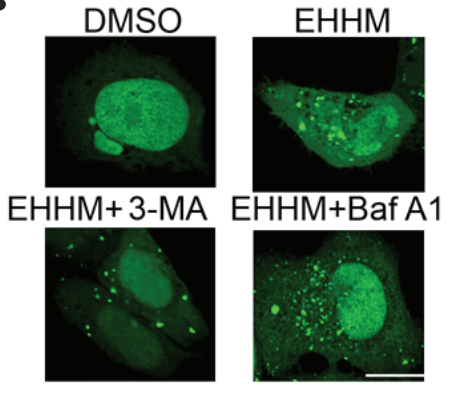

$\mathbf{E}$
D

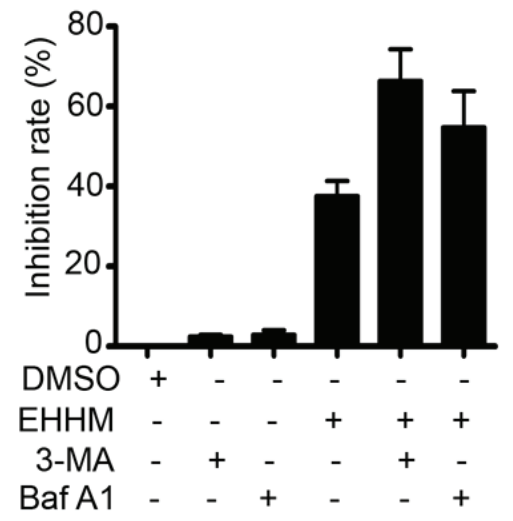

C

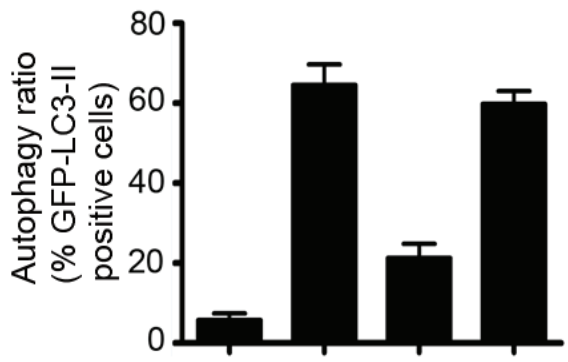<smiles>[Li]</smiles>

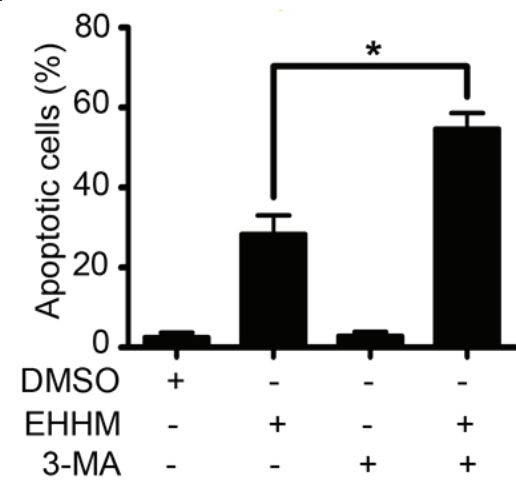

Figure 5. Autophagy inhibition enhances the growth inhibitory and pro-apoptotic effects of EHHM. (A) HepG2 cells were treated with 3-MA or Baf A1, followed by EHHM $(15 \mu \mathrm{M})$ treatment for additional $24 \mathrm{~h}$. Western blot analysis was performed to detect the expression levels of Atg5 and Beclin 1. (B) HepG2 cells transfected with a GFP-LC-3-expressing plasmid were treated with 3-MA or Baf A1, followed by treatment with EHHM (15 $\mu \mathrm{M})$ for additional $24 \mathrm{~h}$. The cells were then observed under a confocal microscope, and (C) the ratios of cells with autophagy formation were calculated. Scale bar, $10 \mu \mathrm{m}$. (D) HepG2 cells were treated with 3-MA or Baf A1, followed by treatment with EHHM $(15 \mu \mathrm{M})$ for additional $24 \mathrm{~h}$, and the cell inhibition rate was then analyzed by 3-(4,5-dimethylthiazol-2-yl)-2,5-diphenyltetrazolium bromide assay. (E) HepG2 cells were treated with 3-MA, followed by EHHM (15 $\mu$ M) treatment for additional $24 \mathrm{~h}$, and the apoptotic cell death rate was analyzed by annexin V/PI staining and flow cytometry. Data represent the mean \pm standard deviation of three independent experiments ("P<0.05). EHHM, E-[6'-(5'-hydroxypentyl)tricosyl]-4-hydroxy-3-methoxycinnamate; GFP, green fluorescent protein; LC3, light chain 3; Atg5, autophagy protein 5; p-, phosphorylated; mTOR, mechanistic target of rapamycin; p70S6K, p70 ribosomal protein S6 kinase; siRNA, small interfering RNA; DMSO, dimethyl sulfoxide; Baf A1, bafilomycin A1; 3-MA, 3-methyl adenine; PI, propidium iodide.

A

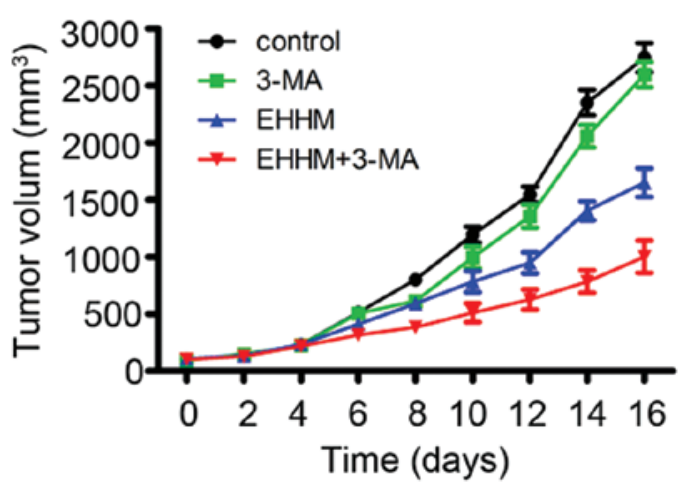

B

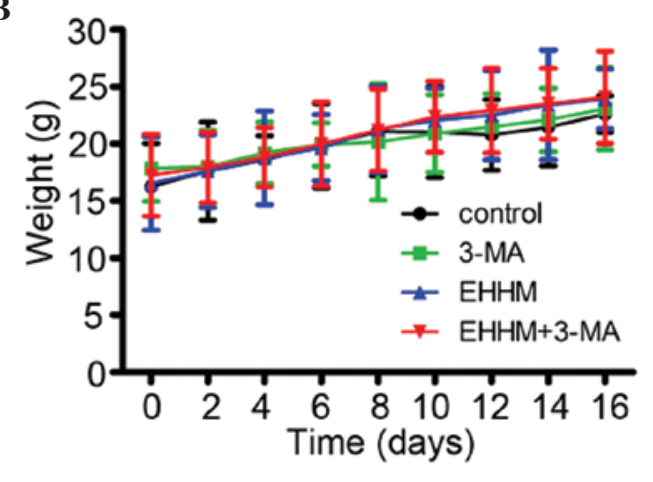

Figure 6. EHHM exhibits antitumor effects on HCC xenograft tumor in vivo. (A) Tumor-bearing mice were treated with 3-MA or EHHM, with or without 3-MA, and the tumor volumes were calculated every 2 days. (B) EHHM with or without 3-MA treatment did not affect the body weight in the murine model. EHHM, E-[6'-(5'-hydroxypentyl)tricosyl]-4-hydroxy-3-methoxycinnamat; 3-MA, 3-methyl adenine.

was also decreased following 3-MA treatment (Fig. 5B and C). Accordingly, 3-MA pretreatment significantly promoted EHHM-induced growth inhibition and apoptotic cell death in HepG2 cells (Fig. 5D and E). Baf A1 treatment markedly promoted the accumulation of GFP-LC3-II autophagic vacuoles in EHHM-treated cells, indicating that Baf A1 inhibited the progression of autophagy by blocking the fusion of autophagosomes with lysosome (Fig. 5B). Similar to 3-MA, Baf A1 pretreatment also significantly promoted EHHM-induced growth inhibition of HepG2 cells (Fig. 5D). These results suggested that EHHM-induced autophagy is a protective response to cell death, and that inhibition of this type of autophagy can enhance the anti-HCC effect of EHHM.

EHHM exhibits antitumor effects on HCC xenograft tumors in vivo. To further evaluate the anti-tumor effect of EHHM 
in vivo, $2.0 \times 10^{6} \mathrm{HepG} 2$ cells suspended in $100 \mu 1$ of serum-free DMEM were subcutaneously inoculated into nude mice to generate xenografted murine models. When the tumors could be measured, the tumor-bearing mice were randomly divided into four groups ( $\mathrm{n}=6 \mathrm{mice} / \mathrm{group}$ ) and administered vehicle control, 3-MA $(24 \mathrm{mg} / \mathrm{kg})$ or EHHM $(50 \mathrm{mg} / \mathrm{kg})$, alone or in combination with 3-MA, every 2 days. As shown in Fig. 6A, the tumor growth was markedly inhibited by EHHM compared with the vehicle control and 3-MA groups, and 3-MA treatment significantly promoted the inhibitory activity of EHHM. By contrast, the above treatments did not significantly reduce the mice body weight, which suggested that EHHM plus 3-MA had no apparent side effects (Fig. 6B). These results preliminarily suggested that EHHM has a potent antitumor activity both in vitro and in vivo, and that the combination of EHHM and autophagy inhibitors provides synergistic anti-proliferative and pro-apoptotic effects in human HCC cells.

\section{Discussion}

A previous study reported that EHHM purified from Livistona chinensis is a key active agent, which exhibited potential anticancer activities towards several cancers, including human myeloid leukemia, HCC and human nasopharyngeal carcinoma (9). HCC is the most common primary cancer in East Asia, particularly in China (47-49), and viral infection, aflatoxin and cirrhosis are the main causes (50). In the present study, the anti-HCC effect and possible mechanisms of EHHM were investigated. The results revealed that EHHM efficiently inhibited the proliferation and induced the apoptosis of HepG2 cells in a time- and dose-dependent manner. In addition, EHHM treatment caused changes in the mitochondrial transmembrane potential, release of cytochrome $c$ from the mitochondria to the cytosol, and activation of the caspase cascade, which indicated that EHHM-induced apoptosis may occur mainly through the activation of the mitochondrial pathway.

Unexpectedly, it was also observed that EHHM treatment induced autophagy formation in HCC cells. EHHM treatment increased the accumulation of autophagic vacuoles, promoted LC3-I/II conversion, and upregulated the expression of several important autophagosome-regulatory genes such as Atg5 and Beclin 1 in HepG2 cells. In fact, accumulating evidence indicated that autophagy could be induced in response to various anticancer treatments in different types of cancer cells, including HCC (51). The $\mathrm{PI} 3 \mathrm{~K} / \mathrm{mTOR} / \mathrm{p} 70$ S6K signaling pathway has been implicated in regulating autophagy and cell death in response to anticancer treatments (43). In the present study, it was also demonstrated that EHHM treatment significantly inhibited the phosphorylation of Akt, mTOR and p70S6K in a time- and dose-dependent manner, which indicated that EHHM-induced autophagy is involved in the inactivation of the Akt/mTOR/p70S6K signaling pathway.

However, the role of autophagy induced by anticancer treatment is not yet fully understood. Currently, there are different perspectives for the role of autophagy in cancer development and chemotherapy (20). Autophagy has been mainly divided into two types depending on its roles for cell fate: One is protective autophagy, which protects cancer cells from death; the other one is autophagic programmed cell death, which can initiate a cell death process in response to anticancer treatments (20). The different roles of autophagy may be related to different cell types and different anticancer therapies (44). Accumulating evidence indicated that protective autophagy may be an important factor that confers cellular resistance to chemotherapy (52). In the present study, the role of EHHM-induced autophagy in cell proliferation and apoptosis in HepG2 cells was investigated. Pretreatment with Atg5 siRNA or autophagy inhibitors (siRNA against Atg5, 3-MA or Baf A1) inhibited EHHM-induced autophagy, and significantly enhanced the EHHM-induced growth inhibitory activity and pro-apoptotic ability, indicating that EHHM-induced autophagy is a protective autophagy. Similarly, several earlier studies have also reported that the use of autophagy inhibitors or siRNAs targeting autophagy-associated genes significantly promoted the apoptosis induced by anticancer treatments in various cancer cells (23,53-55). Therefore, our results, together with previous findings, raise the possibility that there is an unknown crosstalk or feedback network between autophagy and apoptosis in response to anticancer treatments. Furthermore, our study also suggests an attractive strategy for anti-HCC therapy: EHHM in combination with an autophagy inhibitor may have a synergistic effect in HCC chemotherapy.

\section{Acknowledgments}

The authors thank Dr Xiaobin Zeng (Graduate School at Shenzhen, Tsinghua University, Shenzhen, China) for providing EHHM. The present study was supported by the Guangdong Natural Science Foundation (Guangzhou, China; grant no. 2014A030313758), the Doctoral Fund of Ministry of Education of China (Beijing, China; grant no. 20120002120020) and the Science, Technology \& Innovation Commission of Shenzhen Municipality (Shenzhen, China; grant nos. JCYJ20120616213411826 and JCYJ20140417115840285).

\section{References}

1. Chen JG and Zhang SW: Liver cancer epidemic in China: Past, present and future. Semin Cancer Biol 21: 59-69, 2011.

2. Bei CH, Bai H, Yu HP, Yang Y, Liang QQ, Deng YY, Tan SK and Qiu XQ: Combined effects of six cytokine gene polymorphisms and SNP-SNP interactions on hepatocellular carcinoma risk in southern guangxi, china. Asian Pac J Cancer Prev 15: 6961-6967, 2014.

3. Liu HZ, Peng J, Peng CY, Yan M and Zheng F: Glutathione S-transferase M1 null genotype and hepatocellular carcinoma susceptibility in china and india: Evidence from an updated meta-analysis. Asian Pac J Cancer Prev 15: 4851-4856, 2014.

4. Fattovich G, Stroffolini T, Zagni I and Donato F: Hepatocellular carcinoma in cirrhosis: Incidence and risk factors. Gastroenterology 127 (5 Suppl 1): S35-S50, 2004.

5. Bruix J and Sherman M; American Association for the Study of Liver Diseases: Management of hepatocellular carcinoma: An update. Hepatology 53: 1020-1022, 2011.

6. Lin GN, Jiang XM, Peng JW, Xiao JJ, Liu DY and Xia ZJ: Prognostic significance of the peripheral blood absolute monocyte count in patients with locally advanced or metastatic hepatocellular carcinoma receiving systemic chemotherapy. Asian Pac J Cancer Prev 15: 6387-6390, 2014.

7. Cheung S and Tai J: In vitro studies of the dry fruit of Chinese fan palm Livistona chinensis. Oncol Rep 14: 1331-1336, 2005.

8. Kaur G and Singh RP: Antibacterial and membrane damaging activity of livistona chinensis fruit extract. Food Chem Toxicol 46: 2429-2434, 2008. 
9. Zeng X, Wang Y, Qiu Q, Jiang C, Jing Y, Qiu G and He X: Bioactive phenolics from the fruits of Livistona chinensis. Fitoterapia 83: 104-109, 2012.

10. Singh R and Kaur G: Hemolytic activity of aqueous extract of Livistona chinensis fruits. Food Chem Toxicol 46: 553-556, 2008

11. Zhang YX, Abliz G, Ye WJ, Mutalipu Z, Li XW, Wang HQ, Buranjiang $\mathrm{G}$ and Upur $\mathrm{H}$ : Mechanisms of hela cell apoptosis induced by abnormal Savda Munziq total phenolics combined with chemotherapeutic agents. Asian Pac J Cancer Prev 15: 743-747, 2014.

12. Xu H, Tian YN, Dun BY, Liu HT, Dong GK, Wang JH, Lu SS, Chen B and She JX: A novel monoclonal antibody induces cancer cell apoptosis and enhances the activity of chemotherapeutic drugs. Asian Pac J Cancer Prev 15: 4423-4428, 2014

13. Wang CD, Yuan CF, Bu YQ, Wu XM, Wan JY, Zhang L, Hu N Liu XJ, Zu Y, Liu GL and Song FZ: Fangchinoline inhibits cell proliferation via Akt/GSK-3beta/cyclin D1 signaling and induces apoptosis in MDA-MB-231 breast cancer cells. Asian Pac J Cancer Prev 15: 769-773, 2014.

14. Wang YX, Cai H, Jiang G, Zhou TB and Wu H: Silibinin inhibits proliferation, induces apoptosis and causes cell cycle arrest in human gastric cancer MGC803 cells via STAT3 pathway inhibition. Asian Pac J Cancer Prev 15: 6791-6798, 2014.

15. Lowe SW and Lin AW: Apoptosis in cancer. Carcinogenesis 21: 485-95, 2000

16. Chen H, Zeng X, Gao C, Ming P, Zhang J, Guo C, Zhou L, Lu Y, Wang L, Huang L, et al: A new arylbenzofuran derivative functions as an anti-tumour agent by inducing DNA damage and inhibiting PARP activity. Sci Rep 5: 10893, 2015

17. Hsin IL, Ou CC, Wu TC, Jan MS, Wu MF, Chiu LY, Lue KH and Ko JL: GMI, an immunomodulatory protein from Ganoderma microsporum, induces autophagy in non-small cell lung cancer cells. Autophagy 7: 873-882, 2011.

18. Sun J, Zhang C, Bao YL, Wu Y, Chen ZL, Yu CL, Huang YX, Sun Y, Zheng LH, Wang X and Li YX: Parthenolide-induced apoptosis, autophagy and suppression of proliferation in HepG2 cells. Asian Pac J Cancer Prev 15: 4897-4902, 2014.

19. Luo GX, Cai J, Lin JZ, Luo WS, Luo HS, Jiang YY and Zhang Y: Autophagy inhibition promotes gambogic acid-induced suppression of growth and apoptosis in glioblastoma cells. Asian Pac J Cancer Prev 13: 6211-6216, 2012.

20. Mathew R, Karantza-Wadsworth V and White E: Role of autophagy in cancer. Nat Rev Cancer 7: 961-967, 2007.

21. Schweichel JU and Merker HJ: The morphology of various types of cell death in prenatal tissues. Teratology 7: 253-266, 1973.

22. Levine B and Klionsky DJ: Development by self-digestion: Molecular mechanisms and biological functions of autophagy. Dev Cell 6: 463-477, 2004

23. Kanzawa T, Germano IM, Komata T, Ito H, Kondo Y and Kondo S Role of autophagy in temozolomide-induced cytotoxicity for malignant glioma cells. Cell Death Differ 11: 448-457, 2004

24. Cui Q, Tashiro S, Onodera S, Minami M and Ikejima T: Autophagy preceded apoptosis in oridonin-treated human breast cancer MCF-7 cells. Biol Pharm Bull 30: 859-864, 2007.

25. Høyer-Hansen M, Bastholm L, Szyniarowski P, Campanella M, Szabadkai G, Farkas T, Bianchi K, Fehrenbacher N, Elling F, Rizzuto R, et al: Control of macroautophagy by calcium, calmodulin-dependent kinase kinase-beta, and Bcl-2. Mol Cell 25: 193-205, 2007.

26. Lee JY, Lee YM, Chang GC, Yu SL, Hsieh WY, Chen JJ, Chen HW and Yang PC: Curcumin induces EGFR degradation in lung adenocarcinoma and modulates p38 activation in intestine: The versatile adjuvant for gefitinib therapy. PLos One 6: e23756, 2011.

27. Fang HT, Zhang B, Pan XF, Gao L, Zhen T, Zhao HX, Ma L, Xie J, Liu Z, Yu XJ, et al: Bortezomib interferes with C-KIT processing and transforms the $\mathrm{t}(8 ; 21)$-generated. Proc Natl Acad Sci USA 109: 2521-2526, 2012.

28. Lennartsson J, Ma H, Wardega P, Pelka K, Engström U, Hellberg $\mathrm{C}$ and Heldin $\mathrm{CH}$ : The Fer tyrosine kinase is important for platelet-derived growth factor-BB-induced signal transducer and activator of transcription 3 (STAT3) protein phosphorylation, colony formation in soft agar and tumor growth in vivo. J Bio Chem 288: 15736-15744, 2013

29. Zhao YH, Wang T, Yu GF, Zhuang DM, Zhang Z, Zhang HX, Zhao DP and Yu AL: Anti-proliferation effects of interferon-gamma on gastric cancer cells. Asian Pac J Cancer Prev 14: 5513-5518, 2013.

30. Yao G, Yang L, Hu Y, Liang J, Liang J and Hou Y: Nonylphenol-induced thymocyte apoptosis involved caspase-3 activation and mitochondrial depolarization. Mol Immunol 43: 915-926, 2006
31. Yang L,Zhou X, Yang J, Yin X,Han Land Zhao D: Aspirin inhibits cytotoxicity of prion peptide PrP106-126 to neuronal cells associated with microglia activation in vitro. J Neuroimmunol 199: $10-17,2008$

32. Zhang G, Gurtu V, Kain SR and Yan G: Early detection of apoptosis using a fluorescent conjugate of annexin V. Biotechniques 23: 525-531, 1997.

33. Wei H, Li Z, Hu S, Chen X and Cong X: Apoptosis of mesenchymal stem cells induced by hydrogen peroxide concerns both endoplasmic reticulum stress and mitochondrial death pathway through regulation of caspases, p38 and JNK. J Cell Biochem 111: 967-978, 2010.

34. Walsh DM, Tseng BP, Rydel RE, Podlisny MB and Selkoe DJ: The oligomerization of amyloid beta-protein begins intracellularly in cells derived from human brain. Biochemistry 39 10831-10839, 2000.

35. Liu Y, Cao W, Zhang B, Liu YQ, Wang ZY, Wu YP, Yu XJ, Zhang XD, Ming PH, Zhou GB and Huang L: The natural compound magnolol inhibits invasion and exhibits potential in human breast cancer therapy. Sci Rep 3: 3098, 2013.

36. Fernandes-Alnemri T, Litwack G and Alnemri ES: CPP32, a novel human apoptotic protein with homology to Caenorhabditis elegans cell death protein Ced-3 and mammalian interleukin-1 beta-converting enzyme. J Biol Chem 269: 30761-30764, 1994.

37. Nicholson DW, Ali A, Thornberry NA, Vaillancourt JP, Ding CK, Gallant M, Gareau Y, Griffin PR, Labelle M, Lazebnik YA, et al: Identification and inhibition of the ICE/CED-3 protease necessary for mammalian apoptosis. Nature 376: 37-43, 1995.

38. Mizushima N and Komatsu M: Autophagy: Renovation of cells and tissues. Cell 147: 728-741, 2011.

39. He H, Dang Y, Dai F, Guo Z, Wu J, She X, Pei Y, Chen Y, Ling W, Wu C, et al: Post-translational modifications of three members of the human MAP1LC3 family and detection of a novel type of modification for MAP1LC3B. J Biol Chem 278: 29278-29287, 2003.

40. Kabeya Y, Mizushima N, Yamamoto A, Oshitani-Okamoto S, Ohsumi Y and Yoshimori T: LC3, GABARAP and GATE16 localize to autophagosomal membrane depending on form-II formation. J Cell Sci 117: 2805-2812, 2004.

41. Zhong Y,Wang QJ,Li X, Yan Y, Backer JM, Chait BT, Heintz N and Yue Z: Distinct regulation of autophagic activity by Atg14L and rubicon associated with Beclin 1-phosphatidylinositol-3-kinase complex. Nat Cell Biol 11: 468-476, 2009.

42. Pyo JO, Jang MH, Kwon YK, Lee HJ, Jun JI, Woo HN, Cho DH, Choi B, Lee H, Kim JH, et al: Essential roles of Atg5 and FADD in autophagic cell death: Dissection of autophagic cell death into vacuole formation and cell death. J Biol Chem 280: 20722-20729, 2005.

43. Saiki S, Sasazawa Y, Imamichi Y, Kawajiri S, Fujimaki T, Tanida I, Kobayashi H, Sato F, Sato S, Ishikawa K, et al: Caffeine induces apoptosis by enhancement of autophagy via $\mathrm{PI} 3 \mathrm{~K} / \mathrm{Akt} / \mathrm{mTOR} / \mathrm{p} 70 \mathrm{~S} 6 \mathrm{~K}$ inhibition. Autophagy 7: 176-187, 2011

44. White E: Deconvoluting the context-dependent role for autophagy in cancer. Nat Rev Cancer 12: 401-410, 2012

45. Wu YT, Tan HL, Shui G, Bauvy C, Huang Q, Wenk MR, Ong CN, Codogno P and Shen HM: Dual role of 3-methyladenine in modulation of autophagy via different temporal patterns of inhibition on class I and III phosphoinositide 3-kinase. J Biol Chem 285: 10850-10861, 2010.

46. Yamamoto A, Tagawa Y, Yoshimori T, Moriyama Y, Masaki R and Tashiro Y: Bafilomycin A1 prevents maturation of autophagic vacuoles by inhibiting fusion between autophagosomes and lysosomes in rat hepatoma cell line, H-4-II-E cells. Cell Struct Funct 23: 33-42, 1998.

47. Jemal A, Bray F, Center MM, Ferlay J, Ward E and Forman D: Global cancer statistics. CA Cancer J Clin 61: 69-90, 2011.

48. Pourhoseingholi MA, Fazeli Z, Zali MR and Alavian SM: Burden of hepatocellular carcinoma in Iran; Bayesian projection and trend analysis. Asian Pac J Cancer Prev 11: $859-862,2010$.

49. Norsa'adah B and Nurhazalini-Zayani CG: Epidemiology and survival of hepatocellular carcinoma in north-east Peninsular Malaysia. Asian Pac J Cancer Prev 14: 6955-6959, 2013.

50. Arzumanyan A, Reis Hm and Feitelson MA: Pathogenic mechanisms in HBV- and $\mathrm{HCV}$-associated hepatocellular carcinoma. Nat Rev Cancer 13: 123-135, 2013.

51. Kondo Y, Kanzawa T, Sawaya R and Kondo S: The role of autophagy in cancer development and response to therapy. Nat Rev Cancer 5: 726-734, 2005. 
52. Apel A, Herr I, Schwarz H, Rodemann HP and Mayer A: Blocked autophagy sensitizes resistant carcinoma cells to radiation therapy. Cancer Res 68: 1485-1494, 2008

53. Paglin S, Hollister T, Delohery T, Hackett N, McMahill M, Sphicas E, Domingo D and Yahalom J: A novel response of cancer cells to radiation involves autophagy and formation of acidic vesicles. Cancer Res 61: 439-444, 2001.
54. Kanzawa T, Kondo Y, Ito H, Kondo S and Germano I: Induction of autophagic cell death in malignant glioma cells by arsenic trioxide. Cancer Res 63: 2103-2108, 2003.

55. Boya P, González-Polo RA, Casares N, Perfettini JL, Dessen P, Larochette N, Métivier D, Meley D, Souquere S, Yoshimori T, et al: Inhibition of macroautophagy triggers apoptosis. Mol Cell Biol 25: 1025-1040, 2005. 